\title{
Reação de cultivares de soja à Corynespora cassiicola
}

\author{
Adriana Teramoto ${ }^{1}$, Tariane A. Machado ${ }^{1}$, Lucas M. dos Santos ${ }^{1}$, Marcelo R. Volf ${ }^{2}$, Maurício C. Meyer \\ \& Marcos G. da Cunha ${ }^{1}$
}

${ }^{1}$ Setor Fitossanitário, EA/UFG, Cx. Postal 131, 74001-970, Goiânia, GO, Brasil; ${ }^{2}$ Dalcin Planejamento Agrícola, 78690-970, Nova Xavantina, MT; ${ }^{3}$ Embrapa Soja, Cx. Postal 179, 75375-000, Santo Antônio de Góias, GO, Brasil

Autor para correspondência: Adriana Teramoto, e-mail: adritera@terra.com.br

\begin{abstract}
RESUMO
O fungo Corynespora cassiicola, agente causal da mancha-alvo em soja, tem ocorrido com frequência na região Centro-Oeste do Brasil e pode, sob condições de alta temperatura e alta umidade, causar sérios danos à cultura. O uso de cultivares resistentes ao patógeno é recomendado, porém são poucas as disponíveis no mercado. Este trabalho foi realizado visando avaliar a reação de doze cultivares comerciais de soja inoculados com C. cassiicola, em casa de vegetação e no campo. A severidade foi avaliada utilizando escala diagramática de severidade da mancha-alvo. As cultivares menos suscetíveis ao patógeno na casa de vegetação foram BRSGO 7960 e BRS Sambaíba e as mais suscetíveis foram BMX Potência RR e M-SOY 7908 RR. No campo, as cultivares menos suscetíveis foram M-SOY 8866, M-SOY 7908 RR e BMX Potência RR e as mais suscetíveis foram BRSGO 8360 e BRS Tracajá.
\end{abstract}

Palavras-chave: Glycine max, mancha-alvo, resistência genética.

\section{ABSTRACT}

Reaction of soybean cultivars to Corynespora cassiicola

The fungus Corynespora cassiicola, the causal agent of soybean target spot, frequently occurs in the Brazilian midwestern region. Under favorable climate conditions it can cause serious damage to this crop. The use of resistant cultivars is recommended although there are only a few available in the market. This investigation was carried out to evaluate the reaction of twelve soybean commercial cultivars to C. cassiicola. Severity was evaluated using a diagrammatic scale of target spot. The less susceptible cultivars in greenhouse were BRSGO 7960 and BRS Sambaíba and the most susceptible were BMX Potencia RR and M-SOY 7908 RR. In the field, the less susceptible cultivars were M-SOY 8866, M-SOY 7908 RR and BMX Potência RR and the most susceptible were BRSGO 8360 and BRS Tracajá.

Key words: Glycine max, genetic resistance, target spot.

No Brasil, o fungo Corynespora cassiicola (Berk. \& M.A. Curtis) C.T. Wei, agente etiológico da mancha-alvo da soja, infecta toda a parte aérea e o sistema radicular, e vem ocorrendo com frequência nos Cerrados (Almeida et al., 2005). Esse patógeno foi relatado pela primeira vez no Brasil no Mato Grosso em 1974 e no Paraná em 1976 (Almeida et al., 1976). Surtos severos, mas esporádicos, tem sido observados nas regiões mais frias do Sul e nas regiões altas dos Cerrados (Embrapa, 2011).

Várias estratégias são recomendadas para o controle desta doença tais como: o uso de cultivares resistentes, o tratamento de sementes, a rotação de culturas com milho e espécies gramíneas e pulverizações com fungicidas (Embrapa, 2011). Portanto, tornam-se necessários estudos para verificar a resistência das cultivares utilizadas pelos produtores a $C$. cassiicola, provenientes de diferentes regiões do Cerrado.

Desta forma, o presente trabalho teve por objetivo avaliar a reação de cultivares de soja à $C$. cassiicola, em casa de vegetação e no campo.

$O$ ensaio foi realizado na casa de vegetação da Embrapa Transferência de Tecnologia, em Goiânia, GO (16 ${ }^{\circ}$ $40^{\prime} \mathrm{S}$; $\left.49^{\circ} 15^{\prime} \mathrm{O}\right)$ e, no campo, na Fazenda Monte Alegre, em Nova Xavantina, MT ( $14^{\circ} 52^{\prime} 47^{\prime \prime} \mathrm{S} ; 52^{\circ} 22^{\prime}$ 52” O).

Nos ensaios foram utilizados seis isolados de $C$. cassiicola denominados RV01, SO03, QU14, TF07, MO07 e MA01. O isolado RV01 foi proveniente de Rio Verde, GO; SO03 de Sorriso, MT; QU14 de Querência, MT; TF07 de Tasso Fragoso, MA; MO07 de Morrinhos, GO e MA01 de Maracaju, MS. Os isolados foram obtidos de folhas de soja com sintomas típicos da mancha-alvo, isolados primeiramente em meio ágar-água (AA), seguido da repicagem dos bordos do crescimento micelial para batatadextrose-ágar (BDA), sendo posteriormente devidamente preservados pelo método de Castellani (Castellani, 1939, citado por Figueiredo, 1967).

As cultivares de soja utilizadas foram BRSGO 8360; BRSGO 7960; BRSGO 8660; BRS Valiosa RR; BRS Sambaiba; BRS Tracajá; M-SOY 8866; M-SOY 7908 RR; M-SOY 9144 RR; P98Y11; BMX Potência RR e Anta 82 RR. O delineamento experimental utilizado foi fatorial $12 \times 6$, com doze tratamentos (cultivares), seis isolados e quatro repetições, sendo cada uma constituída por um vaso contendo cinco plantas. Os vasos com capacidade de 
5,0 L foram preenchidos com solo utilizado para práticas agrícolas. Quando as plantas de soja atingiram o estádio V8, foram inoculadas por meio de aspersão de suspensão de $10^{4}$ conídios $\mathrm{mL}^{-1}$ de C. cassiicola dos isolados RV01, SO03, QU14, TF07, MO07 e MA01. Estes foram escolhidos dentre os isolados que estavam esporulando abundantemente e a pulverização dos conídios foi realizada até o ponto de escorrimento superficial das folhas. Após a inoculação, os vasos foram mantidos por 48 horas em câmara úmida, cobertos com lona plástica transparente.

A severidade (\% de área foliar lesionada) da doença foi avaliada na folha mais infectada da planta, quando estas estavam no estádio R5.4, utilizando a escala diagramática de Soares et al. (2009). Os dados obtidos foram submetidos à análise de variância utilizando o programa Estat (Estat, 1994) e, quando significativos, as médias dos tratamentos foram separadas pelo teste de Tukey a 5\% de probabilidade.

No experimento de campo as cultivares de soja utilizadas foram as mesmas testadas em casa de vegetação, diferindo apenas na utilização de um único isolado inoculado, o SO03, proveniente da mesma região onde foi instalado este ensaio. Cada parcela media $10 \mathrm{~m}^{2}$, sendo 4 linhas de $5 \mathrm{~m}$ e espaçamento entre linhas de $0,5 \mathrm{~m}$, com 4 repetições. As plantas foram inoculadas quando atingiram o estádio R1, na concentração de $10^{4}$ conídios $\mathrm{mL}^{-1}$, até o ponto de escorrimento superficial das folhas.
A severidade (\% de área com sintoma) da doença foi avaliada em R5.1, R5.3 e R5.4, utilizando-se escala diagramática elaborada e validada por Soares et al. (2009). Os dados obtidos foram submetidos à análise de variância e as médias foram agrupadas pelo teste de Scott-Knott a 5\% de probabilidade (Canteri et al., 2001).

As cultivares de soja apresentaram reações diferenciais frente aos seis isolados de C. cassiicola. Foi possível observar maiores severidades de mancha-alvo nas cultivares inoculadas com o isolado $\mathrm{SO} 03$ proveniente de Sorriso, MT (2,80 a 11,57\%), Morrinhos (MO07) (2,10 a $6,70 \%)$ e de Rio Verde (RV01) $(0,66$ a 7,0\%). A cultivar BRS 7960 dentre as cultivares, foi a mais resistente ao isolado RV01 e a cultivar M-SOY 8866 foi a mais suscetível ao isolado SO03. Para o isolado QU14, somente BRS Sambaíba foi menos suscetível. Para o isolado TF07, as cultivares BRS 8660, M-SOY 9144 RR, M-SOY 8866, P98Y11, BRS 7960 e BRS Sambaíba foram consideradas resistentes. Para o isolado MA01, todas as cultivares foram consideradas resistentes (Tabela 1).

Nas cultivares BRSGO 8360, M-SOY 9144 RR e a P98Y11 ocorreu maior severidade de mancha-alvo quando inoculadas com o isolado SO03 proveniente de Sorriso. A cultivar M-SOY 7908 RR apresentou alta severidade quando inoculada com os isolados RV01 e SO03. A cultivar BMX Potência RR apresentou alta severidade quando

TABELA 1 - Severidade (\% de área foliar com sintomas) de mancha-alvo causada por isolados de Corynespora cassiicola, em cultivares de soja no estádio R5.4 em casa de vegetação em Goiânia, e em campo inoculadas com apenas um isolado, em Nova Xavantina, 2012

\begin{tabular}{|c|c|c|c|c|c|c|c|}
\hline \multirow[t]{3}{*}{ Cultivares } & \multirow{2}{*}{\multicolumn{6}{|c|}{$\begin{array}{c}\text { Severidade (\%) } \\
\text { Casa de vegetação }{ }^{1}\end{array}$}} & \multirow{3}{*}{$\begin{array}{c}\text { Campo }^{2} \\
\text { SO03 }\end{array}$} \\
\hline & & & & & & & \\
\hline & RV01 & SO03 & QU14 & TF07 & MO07 & MA01 & \\
\hline BRSGO 8360 & $2,15 \mathrm{bcdBC}^{3}$ & $6,42 \mathrm{bA}$ & $1,75 \mathrm{abBC}$ & $3,46 \mathrm{abABC}$ & $3,90 \mathrm{abAB}$ & $1,35 \mathrm{aC}$ & $13,8 a^{4}$ \\
\hline BRS 7960 & $0,66 \mathrm{dBC}$ & $4,57 \mathrm{bA}$ & $1,55 \mathrm{abBC}$ & $1,34 \mathrm{bBC}$ & $3,07 \mathrm{abAB}$ & $1,50 \mathrm{aBC}$ & $7,0 \mathrm{~b}$ \\
\hline BRS 8660 & $0,98 \mathrm{cdB}$ & $2,84 \mathrm{bAB}$ & $2,10 \mathrm{abAB}$ & $1,75 \mathrm{bAB}$ & $2,97 \mathrm{bA}$ & $1,95 \mathrm{aAB}$ & $5,0 \mathrm{~b}$ \\
\hline BRS Valiosa RR & $2,40 \mathrm{bcdAB}$ & $4,55 \mathrm{bA}$ & $1,90 \mathrm{abAB}$ & $3,50 \mathrm{abAB}$ & $3,75 \mathrm{abA}$ & $1,30 \mathrm{aB}$ & $8,0 \mathrm{~b}$ \\
\hline BRS Sambaiba & $2,36 \mathrm{bcdAB}$ & $3,07 \mathrm{bA}$ & $0,90 \mathrm{bB}$ & $1,22 \mathrm{bAB}$ & $1,95 \mathrm{bAB}$ & $1,15 \mathrm{aAB}$ & $6,3 b$ \\
\hline BRS Tracajá & $2,79 \mathrm{bcAB}$ & $5,02 \mathrm{bA}$ & $3,62 \mathrm{abA}$ & $3,25 \mathrm{abAB}$ & $4,10 \mathrm{abA}$ & $1,40 \mathrm{aB}$ & $14,0 \mathrm{a}$ \\
\hline M-SOY 8866 & 1,73 bcdA & $2,8 \mathrm{bA}$ & $1,32 \mathrm{abA}$ & $1,58 \mathrm{bA}$ & $2,10 \mathrm{bA}$ & $1,70 \mathrm{aA}$ & $2,5 \mathrm{c}$ \\
\hline M-SOY 7908 RR & $7,00 \mathrm{aAB}$ & $11,57 \mathrm{aA}$ & $2,80 \mathrm{abCD}$ & $2,73 \mathrm{abCD}$ & 4,60 abBC & $1,25 \mathrm{aD}$ & $1,8 \mathrm{c}$ \\
\hline M-SOY 9144 RR & $1,81 \mathrm{bcdB}$ & $5,42 \mathrm{bA}$ & $2,60 \mathrm{abAB}$ & $1,64 \mathrm{bB}$ & $3,30 \mathrm{abAB}$ & $1,25 \mathrm{aB}$ & $5,3 \mathrm{~b}$ \\
\hline P98Y11 & $3,47 \mathrm{abAB}$ & $5,92 \mathrm{bA}$ & $1,70 \mathrm{abBC}$ & $1,47 \mathrm{bBC}$ & $4,80 \mathrm{abA}$ & $1,20 \mathrm{aC}$ & $5,3 \mathrm{~b}$ \\
\hline BMX Potência RR & $4,00 \mathrm{abAB}$ & $5,12 \mathrm{bA}$ & $1,87 \mathrm{abBC}$ & $5,42 \mathrm{aA}$ & $6,70 \mathrm{aA}$ & $1,40 \mathrm{aC}$ & $4,0 \mathrm{c}$ \\
\hline Anta 82 RR & $2,69 \mathrm{bcdA}$ & $3,37 \mathrm{bA}$ & $2,30 \mathrm{abA}$ & $2,62 \mathrm{abA}$ & $4,05 \mathrm{bA}$ & $1,55 \mathrm{aA}$ & $5,5 \mathrm{c}$ \\
\hline Média & $2,67 \mathrm{C}$ & $5,06 \mathrm{~A}$ & $2,03 \mathrm{CD}$ & $2,50 \mathrm{C}$ & $3,45 \mathrm{~B}$ & $1,42 \mathrm{D}$ & 6,54 \\
\hline
\end{tabular}

${ }^{1}$ Ensaio em casa de vegetação realizado em Goiânia, GO. ${ }^{2}$ Ensaio no campo realizado em Nova Xavantina, MT. ${ }^{3}$ Médias seguidas de mesma letra minúscula na coluna e letras maiúsculas na linha não diferem entre si pelo teste de Tukey a 5\% de significância, em casa de vegetação. Médias de quatro repetições. ${ }^{4}$ Médias seguidas de mesma letra minúscula na coluna pertencem ao mesmo grupamento pelo teste de Scott-Knott a $5 \%$ de significância, em campo. 
inoculada com os isolados provenientes de Sorriso (SO03), Tasso Fragoso (TF07) e Morrinhos (MO07).

De acordo com a classificação de resistência à mancha-alvo apresentada pela Embrapa Soja, somente a cultivar M-SOY 7908 RR é classificada como medianamente resistente (MR) e as demais são todas resistentes, quando inoculadas com o isolado RV01. Já para o isolado SO03, as cultivares M-SOY 7908 RR, M-SOY 9144 RR, P98Y11 e BMX Potência RR são medianamente resistentes. Todas as cultivares inoculadas com os isolados QU14 e MA01 foram classificadas como resistentes. Apenas a cultivar BMX Potência RR foi considerada medianamente suscetível, quando inoculada com os isolados TF07 e MO07.

A partir destes resultados observou-se variação das reações das cultivares aos diferentes isolados, o que provavelmente reflete a especificidade da resistência frente a variabilidade do patógeno. Existem outros processos de variabilidade genética que podem ocorrer. Com relação ao estudo da variabilidade genética de Corynespora cassiicola, há trabalho que tentou relacionar origem do hospedeiro com a virulência (Silva et al., 2003), a especialização do hospedeiro com a diversidade filogenética (Dixon et al.,2009), a morfologia com a análise molecular dos isolados de diferentes hospedeiros (Qi et al., 2011) e, a patogenicidade com a variação genética dos isolados (Shimomoto et al., 2011).

Dixon et al. (2009) observaram que seis linhagens filogenéticas de $C$. cassiicola tiveram correlação com a origem do hospedeiro, com patogenicidade e com taxa de crescimento, mas não apresentaram correlação com a localização geográfica. Ainda, segundo os autores, genótipos de $C$. cassiicola foram amplamente distribuídos geograficamente, indicando dispersão a longa distância e global de linhagens clonais. Desta forma, quanto a este trabalho, há necessidade de elucidar a variabilidade genética dos isolados para poder explicar melhor os resultados.

Um outro fator que pode ter levado a variação dos resultados é a padronização do ambiente na casa de vegetação, que pode ter interferido na agressividade dos isolados, causando reação diferente da observada nos campos de produção. Segundo Vida et al. (2004), a elevada umidade do ar associada às altas temperaturas propiciam condições para algumas doenças da parte aérea tornaremse muito mais severas nos cultivos em estufa que nos convencionais.

Assim, pode-se concluir que dentre as cultivares de soja testadas não foi observado resistência aos isolados de C. cassiicola, porém, houve cultivares que apresentaram menores severidades de mancha-alvo. Essas variedades apresentam alto potencial para serem indicadas ao cultivo em regiões de alta favorabilidade para mancha-alvo.

Os resultados de casa de vegetação e do campo diferiram possivelmente devida às condições ambientais $\mathrm{e}$ pela utilização no campo de apenas um isolado inoculado. As cultivares mais suscetíveis em campo foram a BRSGO 8360 e a BRS Tracajá. A cultivar BRSGO 8360 é semiprecoce e indicada para plantio nos estados de Goiás, Distrito Federal, Minas Gerais, Mato Grosso do Sul e Mato Grosso. A cultivar BRS Tracajá é de ciclo médio e indicada para os estados do Maranhão, Tocantins, Piauí, Roraima e Pará.

Ainda no experimento de campo as cultivares medianamente suscetíveis foram a BRSGO 7960, BRSGO 8660, BRS Valiosa RR, BRS Sambaíba, M-SOY 9144 RR, P98Y11 e a Anta 82 RR, e as menos suscetíveis a M-SOY 8866, M-SOY 7908 RR e a BMX Potência RR.

Miguel-Wruk et al. (2011) observaram que nas regiões de Sorriso e Sinop (Estado do Mato Grosso) as cultivares Anta 82 RR, M-SOY 7908 RR e BRS Valiosa RR comportaram-se como resistentes a C. cassiicola, apresentando severidade inferior a 3\%. Segundo os autores, as cultivares Anta $82 \mathrm{RR}$ apresentou severidade de $5,5 \%$ e a BRS Valiosa RR de $8 \%$, ambas consideradas medianamente resistentes.

Quando se comparada as reações observadas em casa de vegetação e no campo, as cultivares BMX Potência RR e M-SOY 7908 RR comportaram-se como mais suscetíveis na casa de vegetação, porém foram mais resistentes no campo. Um dos motivos para esse resultado contrastante pode ser devido a inoculação de diversos isolados na casa de vegetação, expondo as cultivares a maior diversidade de isolados. Outro fator ocorrido seria escape a C. cassiicola como: alguns cultivares mais precoces e ambiente diferente daquele da casa de vegetação.

\section{AGRADECIMENTOS}

Os autores agradecem à Coordenação de Aperfeiçoamento de Pessoal de Nível Superior - CAPES, pela bolsa de pós-doutorado concedida a A. Teramoto.

\section{REFERÊNCIAS BIBLIOGRÁFICAS}

Almeida AMR, Ferreira LP, Yorinori JT, Silva JFV, Henning AA, Godoy CV, Costamilan LM, Meyer MC (2005) Doenças da soja. In: Kimati H, Amorim L, Rezende JAM, Bergamin Filho A, Camargo LEA (Eds.). Manual de Fitopatologia - Vol. 2. Doenças de Plantas Cultivadas. 4. ed. São Paulo SP. Editora Agronômica Ceres. pp. 570-588.

Almeida AMR, Machado CC, Ferreira LP, Lehman PS, Antonio H (1976) Ocorrência de Corynespora cassiicola (Berk. \& Curt.) Wei no estado de São Paulo. Fitopatologia Brasileira 1:111-112.

Anônimo (2011) Tecnologias de Produção de Soja, Região Central do Brasil 2012 e 2013. Londrina PR. Embrapa Soja.

Canteri MG, Althaus RA, Virgens Filho JS, Giglioti EA, Godoy CV (2001) SASM - Agri: Sistema para análise e separação de médias em experimentos agrícolas pelos métodos Scott-Knot, Tukey e Duncan. Revista Brasileira de Agrocomputação 1:18-24.

Dixon LJ, Schlub RL, Pernezny K, Datnoff E (2009) Host specialization and phylogenetic diversity of Corynespora cassiicola. Phytopathology 99:1015-1027.

Embrapa (2011) Tecnologias de Produção de Soja, Região Central do Brasil 2012 e 2013. Londrina PR. Embrapa Soja. 
Estat (1994) Sistema para análises estatísticas (v. 2.0). Jaboticabal SP. Departamento de Ciências Exatas, FCAV-UNESP.

Figueiredo MB (1967) Estudos sobre a aplicação do método de Castellani para conservação de fungos patógenos em plantas. $\mathrm{O}$ Biológico 33:9-13.

Miguel-Wruck DS, Paes JMV, Zito RK, Wruk FJ, Damasceno AG, Moulin MC (2011) Seleção de linhagens de soja quanto a resistência de Corynespora cassiicola safras 2009/2010. XXXII Reunião de Pesquisa de Soja da Região Central do Brasil, Resumos... São Pedro SP. pp. 120-122.

Qi YX, Zhang X, Pu JJ, Liu XM, Lu Y, Zhang H, Zhang HQ, Lv YC, Xie YX (2011) Morphological and molecular analysis of genetic variability within isolates of Corynespora cassiicola from different hosts. European Journal of Plant Pathology 130:83-95.
Shimomoto Y, Sato T, Hojo H, Morita Y, Takeuchi S, Mizumoto H, Kiba A, Hikichi Y (2011) Pathogenic and genetic variation among isolates of Corynespora cassiicola in Japan. Plant Pathology 60:253-260.

Silva WPK, Karunanayake EH, Wijesundera RLC, Priyanka UMS (2003) Genetic variation in Corynespora cassiicola: A possible relationship between host origin and virulence. Mycological Research 107:567-571.

Soares RM, Godoy CV, Oliveira MCN (2009) Escala diagramática para avaliação da severidade da mancha alvo da soja. Tropical Plant Pathology 34:333-338.

Vida JB, Zambolim L, Tessmann DJ, Brandão Filho JUT, Verzignassi JR, Caixeta MP (2004) Manejo de doenças de plantas em cultivo protegido. Fitopatologia Brasileira 29:355-372. 